\title{
FACTORS ASSOCIATED WITH HYPERTENSION PREVALENCE AND CONTROL AMONG LEBANESE TYPE 2 DIABETIC PATIENTS
}

\author{
LAMA SOUBRA*, HANAN NUREDDIN, AMAL GALAL OMAR, MOUNZER SALEH
}

\author{
Pharmacy Practice Department, Beirut Arab University \\ Email: 1.soubra02@bau.edu.lb
}

Received: 06 Jun 2016 Revised and Accepted: 12 Aug 2016

\begin{abstract}
Objective: The objectives of this study were to assess the prevalence of hypertension and the level of blood pressure (BP) control among a cohort of diabetic Lebanese patients on antihypertensive medications, as well as to identify factors associated with hypertension prevalence and uncontrolled BP.

Methods: This cross-sectional retrospective study was conducted in a tertiary health care clinic that is specialized in the management and follow-up of diabetic outpatients.

Results: Among the 700 type 2 diabetes mellitus patient files that were screened529 (75\%) were found to have hypertension. Hypertension was more prevalent in women, patients aged $\geq 65$-year-old, and those having a body mass index (BMI) $\geq 070\left(\mathrm{~kg} / \mathrm{m}^{2}\right)$ (p-value $\left.<0.05\right)$. Among the hypertensive cohort, 465 T2DM were on antihypertensive medications and were included in the hypertension control analysis. Ninety-three patients $(20 \%)$ attained $\mathrm{BP}$ control $(\mathrm{SBP}<140$ and $\mathrm{DBP}<90 \mathrm{mmHg})$. Multivariate analyses revealed three factors that were significantly associated with uncontrolled BP control: Age being $\geq 65$ y (adjusted OR $=1.96$ (95\% CI: 1.07-3.61, p-value $<0.05$ ), male gender (adjusted OR $=2.57,95 \% \mathrm{CI}$ : 1.41-4.66, p-value $<0.05$ ) and uncontrolled HDL (adjusted OR $=1.58,95 \% \mathrm{CI}: 1.33-2.01$, p-value $=0.05$ ).
\end{abstract}

Conclusion: Hypertension is prevalent among the study patients. However, attainment of BP control was poor among these patients. Therefore, there is a need for studies that determine reasons behind this low BP control rate in order to design interventions aiming at improving the standard of care for these patients.

Keywords: Diabetes Mellitus Type II, Hypertension risk factors, Hypertension prevalence, Blood pressure control, Lebanon

(C) 2016 The Authors. Published by Innovare Academic Sciences Pvt Ltd. This is an open access article under the CC BY license (http://creativecommons. org/licenses/by/4. 0/) DOI: http://dx.doi.org/10.22159/ijpps.2016v8i10.13298

\section{INTRODUCTION}

Type 2 Diabetes Mellitus (T2DM) and hypertension (HT) are common chronic disorders that often coexist [1]. Large epidemiological studies showed that the coexistence of elevated Blood Pressure (BP) in patients with T2DM is associated with increased risk of mortality and morbidity because of cardiovascular complications [2-5]. Left ventricular hypertrophy and coronary artery disease were reported to be more common in diabetic hypertensive patients than in patients suffering from hypertension or diabetes alone [2]. Furthermore, it was reported that men with hypertension and T2DM have a $66 \%$ higher risk of suffering a stroke or heart attack than men who only have hypertension [5]. In these patients, a difference of $5 \mathrm{mmHg}$ in either systolic blood pressure (SBP) or diastolic blood pressure (DBP) increases the risk of cardiovascular events or death by 20 to $30 \%$ [6]

Benefits of adequately controlling BP in diabetic patients have been documented by numerous studies [2, 7-9]. The action to control cardiovascular risk in diabetes (ACCORD) trial showed that patients with diabetes whose SBP ranged between 130 to $140 \mathrm{~mm}$ Hgwere 46\% less likely to die from any cause or suffer stroke or a nonfatal myocardial infarction (MI) [10]. Moreover, the hypertension optimal treatment (HOT) trial confirmed a 51\% reduction in major cardiovascular events in the study group allocated to a target DBP of $\leq 80 \mathrm{~mm} \mathrm{Hg}$ when compared with the group with a DBP of $\leq 90 \mathrm{~mm} \mathrm{Hg}$ [11]. In the UK prospective study group 36, each $10 \mathrm{~mm} \mathrm{Hg}$ decrease in SBP from baseline resulted in a $19 \%$ decrease in the risk of diabetesrelated mortality, a $13 \%$ in allcause mortality and a $13 \%$ decrease in MI [12]. BP control in patients with T2DM without overt albuminuria has also been shown to stabilize kidney function over a 5-year period [13].

Therefore, controlling BP in T2DM patients and maintaining this BP control throughout patient follow-up has been strongly recommended and in 2013, a target BP for T2DM patients was set at $<140 / 90 \mathrm{~mm} \mathrm{Hg}$ by the American diabetes association (ADA) [14].
Despite these recommendations which were based on the known benefits of lowering BP in preventing or slowing cardiovascular diseases in T2DM patients, studies consistently demonstrate that most diabetic patients do not achieve recommended levels of BP control and the majority have a $\mathrm{BP}>140 / 90 \mathrm{~mm} \mathrm{Hg}[15,16]$.

In Lebanon, the prevalence of diabetes is estimated to range from 8.5\% to $15.8 \%$ among adults aged $>25 \mathrm{y}$ and according to the world health organisation (WHO) the number of people with diabetes is expected to significantly increase during the coming years $[17,18]$. Moreover, two previous studies showed that among hypertensive Lebanese patients $23.9 \%$ and $27 \%$ were diabetics respectively [16-19] and that more than half of the hypertensive diabetics had uncontrolled BP [19]. There are scant data on the prevalence of hypertension in T2DM Lebanese patients, their status concerning BP control and factors affecting it. This information is highly needed to help manage cardiovascular risk factors and delay complications in these patients. Therefore, the present study was conducted with the aims of: (1) assessing the prevalence of high BP (i.e. SBP $\geq 140$ and/or DBP $\geq 90 \mathrm{~mm} \mathrm{Hg}$ ) in a cohort of T2DM Lebanese patients, (2) determining the prevalence of controlled $\mathrm{BP}(\mathrm{SBP}<140 \mathrm{~mm}$ $\mathrm{Hg}$ and $\mathrm{DBP}<90 \mathrm{~mm} \mathrm{Hg}$ ) among this cohort under current clinical practice and (3) identifying factors associated with uncontrolled BP (i.e. $\mathrm{SBP}>140$ and/or DBP $>90 \mathrm{~mm} \mathrm{Hg}$ ).

\section{MATERIALS AND METHODS}

\section{Methods}

The study was approved by the ethical committee of the Faculty of Pharmacy at Beirut Arab University as well as by the director of the clinic where the study was conducted. Ethical approval from a review board was not required since this is a descriptive retrospective study. All patient data were handled and processed in accordance to the recommendations of good clinical practice.

\section{Design and setting}

This cross-sectional retrospective study was conducted in a tertiary health care clinic that is specialized in the management and follow- 
up of diabetic outpatients. The clinic which is located in Beirut city serves patients from across all regions of Lebanon(mainly from greater Beirut and Mount Lebanon regions that are home to $53.6 \%$ of the Lebanese population) and from a variety of socioeconomic backgrounds (mainly middle to high).

\section{Data collection}

Medical records of all patients who received care between January 2013 and December 2013 were consecutively screened to identify those with T2DM diagnosis as documented by the treating physician. Medical records of pregnant women patients, patients aged $\leq 18 \mathrm{y}$ and those who did not authorize the use of their data were excluded from the study.

Data that were collected from the selected medical records included the followings: patient demographics (age and gender), social habits (smoking status and alcohol use), body mass index (BMI) and comorbid conditions. A person was considered a non-smoker when the variable had responses of "no" or "ex-smoker".

They also included DM related information which consisted of the presence of a family history of DM (i.e. presence of diabetes in any paternal or maternal relative), diabetes duration, presence of diabetes complications, fasting blood sugar (FBS) level, glycated Hemoglobin (HbA1c) level and antidiabetic medications. BP readings, prescribed antihypertensive (type and duration) and anti-platelet medications as well as patient lipid profile were also obtained.

All data were collected at the last clinic visit during the study period.

Lab data were categorized based on their control status prior analyzing the factors that were associated with uncontrolled BP. This was done using the following cut off points: fasting blood sugar (FBS) $<70-130 \mathrm{mg} / \mathrm{dl}$, glycated hemoglobin $(\mathrm{HbA1C}$ ) value of $<63$ $\mathrm{mmol} / \mathrm{l}(<7 \%)$, low density lipoprotein cholesterol (LDL-C) 1.8102 $\mathrm{mmol} / \mathrm{l}(70 \mathrm{mg} / \mathrm{dl})$ for T2DM patients with overt cardiovascular diseases or $2.586 \mathrm{mmol} / \mathrm{l}(100 \mathrm{mg} / \mathrm{dl})$ for those without overt cardiovascular diseases, high density lipoprotein cholesterol (HDLC) $>1.034 \mathrm{mmol} / \mathrm{l}(40 \mathrm{mg} / \mathrm{dl})$ in men, and at $>1.293 \mathrm{mmol} / \mathrm{l}(50$ $\mathrm{mg} / \mathrm{dl}$ ) in women, serum triglycerides (TG) $<1.693 \mathrm{mmol} / \mathrm{l}(150$ $\mathrm{mg} / \mathrm{dl}$ ) and total cholesterol (TC) $<5.172 \mathrm{mmol} / \mathrm{l}(200 \mathrm{mg} / \mathrm{dl})$ [14].

Patients were initially grouped into two groups, those "with hypertension" and those "without hypertension". This was done using either a documented clinical diagnosis of hypertension in the medical record or the use of antihypertensive medications for lowering BP. The cut-off values used by the treating physician for hypertension diagnosis were a SBP $>140 \mathrm{~mm} \mathrm{Hg}$ and/or a DBP $>90 \mathrm{~mm} \mathrm{Hg}$ in three or more consecutive clinic visits, for those who were diagnosed during the year 2013 or a $\mathrm{SBP}>130 \mathrm{~mm} \mathrm{Hg}$ and/or a $\mathrm{DBP}>80 \mathrm{~mm} \mathrm{Hg}$ for those who were diagnosed prior that year. Patients in the "with hypertension" group were further divided into two subgroups: those with "controlled BP" (i.e. patients having SBP $<140 \mathrm{~mm} \mathrm{Hg}$ and/or $\mathrm{DBP}<90 \mathrm{~mm} \mathrm{Hg}$ ) and those with uncontrolled BP (i.e. patients having SBP $\geq 140 \mathrm{~mm} \mathrm{Hg}$ or DBP $\geq 90 \mathrm{~mm} \mathrm{Hg}$ ). This was done using the means of two SBP and DBP readings obtained in the last clinic visit, taken 5 min apart by a trained nurse and comparing them to the 2013 ADA BP goals [14]. Patients who had no documented BP readings were excluded from this analysis.

Data were analyzed to identify firstly factors associated with hypertension prevalence and secondly factors associated with uncontrolled BP. The model with hypertension prevalence as outcome variable included the following covariates: age, gender, BMI, self-reported smoking status, alcohol use, diabetes mellitus duration and presence of dyslipidemia as a co-morbid condition.

The model with controlled BP as outcome variable included all retrieved co-variates/predictor variables.

\section{Statistical analysis}

Data were entered and analyzed using the Statistical Package for Social Sciences (SPSS), version 21. Categorical variables were summarized by calculating the number and percent, whereas the continuous ones were summarized by calculating the mean and standard deviation. The chi-square test was used to assess the association between study outcome and the different categorical variables, whereas independent sample t-test was used for continuous ones. For categorical variables, odds ratios (OR) and 95\% confidence interval (CI) were calculated.

Multivariate logistic regression analysis was carried out to identify independent factors that were associated with hypertension prevalence and those associated with uncontrolled BP. Adjusted OR and $95 \% \mathrm{CI}$ were reported. A p-value of $\leq 0.05$ was considered to be statistically significant.

\section{RESULTS}

One thousand fifty patients received care during the study period out of which 715 had T2DM. Fifteen patients were excluded from the study because either they were pregnant (10) or they refused authorization for use of their medical records (5). Of the 700 T2 DM, $529(75.5 \%)$ were hypertensives and $171(24.5 \%)$ patients were not hypertensives.

Moreover, of the hypertensive group, 64 hypertensive patients had no documentation of their BP readings and were therefore excluded from BP control analysis. The final sample consisted of 465 hypertensive patients (fig. 1).

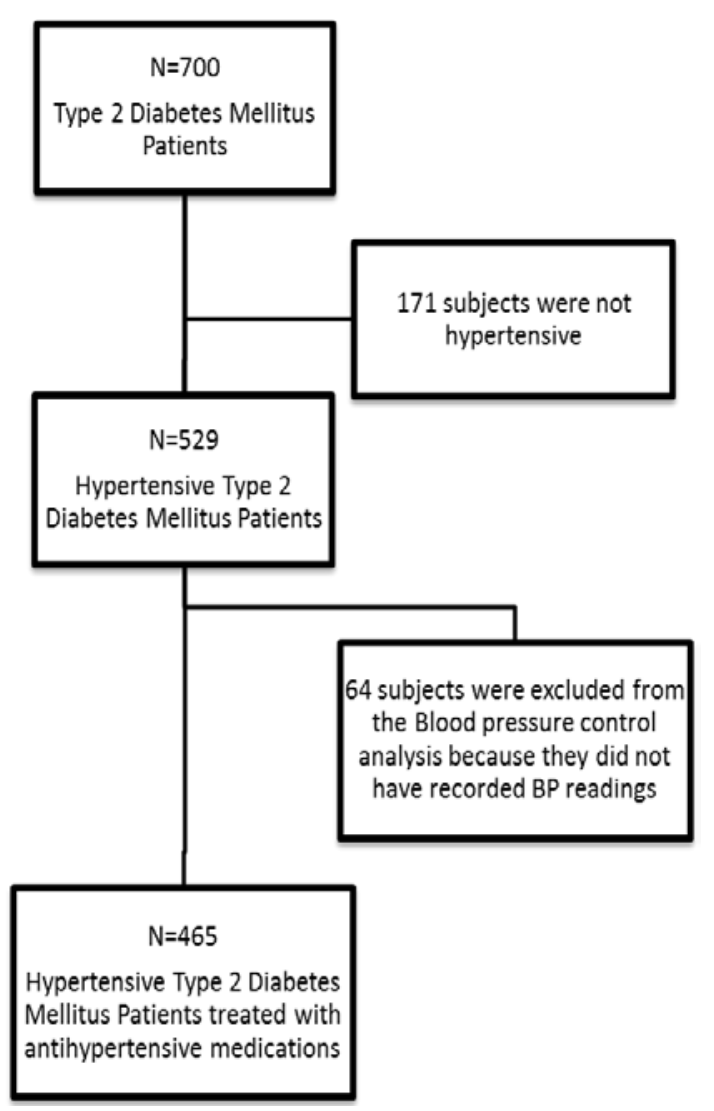

Fig. 1: Study sample

\section{Hypertensive cohort}

Table 1 shows that T2DM patients who were hypertensive had an average age of $61.4 \pm 11.1 \mathrm{y}$. Forty percent were aged above $65 \mathrm{y}$ and $48 \%$ were females. Eighty-nine percent were overweight and obese and $56 \%$ were smokers. They had a mean SBP of $144.5 \pm 9.4 \mathrm{~mm} \mathrm{Hg}$ and a mean DBP of $79.7 \pm 5.6 \mathrm{~mm} \mathrm{Hg}$. Seventy-five percent of recently diagnosed patients were hypertensive at the time of diagnosis. Sixtythree percent of patients were on two or more antihypertensive agents. The most commonly prescribed antihypertensive agents were angiotensin converting enzyme inhibitors (ACEIs) and angiotensin receptor blockers (ARBs) accounting for $78.7 \%$ of the 
total antihypertensive prescriptions, followed by beta-blockers (49.5\%) and diuretics (40.2\%) (table 4).

Sixty-three percent of the patients were receiving antiplatelet therapy either as monotherapy (58.1\%) or as dual therapy (5.2\%) (table 4). The most commonly prescribed antiplatelet therapy was acetylsalicylic acid (46.7\%) (table 4). Multivariate analysis showed that hypertension was more prevalent in female T2DM patients than male patients (OR=1.43, 95\% CI: $1.15-3.22$, p-value $<0.05$ ), in patients aged $\geq 65$-old, (OR $=3.23,95 \% \mathrm{CI}: 1.62-14.74$, p-value $<0.05)$, obese $(\mathrm{OR}=1.32,95 \% \mathrm{CI}: 1.1-2.30$, p-value $<0.05))$ and in those having dyslipidemia $\quad(\mathrm{OR}=4.79, \quad 95 \% \quad \mathrm{CI}: \quad 3.43-6.68, \quad \mathrm{p}$-value<0.05). Hypertension prevalence was not found to be significantly associated with the duration of diabetes mellitus ( $p$-value $>0.05$ ).

Table 1: Association between hypertension prevalence and demographic, social habits and health status among the cohort of type 2 diabetes mellitus Lebanese patients

\begin{tabular}{|c|c|c|c|c|c|c|c|c|}
\hline \multicolumn{2}{|l|}{ Variables } & $\begin{array}{l}\text { Total } \\
N(\%) \\
N=700\end{array}$ & $\begin{array}{l}\text { No hypertension } \\
\mathrm{N}(\%) \\
\mathrm{N}=171\end{array}$ & $\begin{array}{l}\text { hypertension } \\
N(\%) \\
N=529\end{array}$ & OR (95\% CI) & P-value & $\begin{array}{l}\text { Adjusted OR } \\
(95 \%)\end{array}$ & $\begin{array}{l}\text { P- } \\
\text { value }\end{array}$ \\
\hline \multirow[t]{2}{*}{ Age (years) } & Mean (sd) & $58.5(12.3)$ & $51.0(11.6)$ & $60.9(11.6)$ & & 0.001 & & \\
\hline & $\geq 65$ & $\begin{array}{l}223 \\
(31.9 \%)\end{array}$ & $16(9.4 \%)$ & $207(39.1 \%)$ & $\begin{array}{l}6.23(3.62- \\
10.72)\end{array}$ & 0.001 & $\begin{array}{l}3.23(1.62- \\
14.74)\end{array}$ & 0.03 \\
\hline \multicolumn{2}{|l|}{ Male Gender } & $\begin{array}{l}405 \\
(57.9 \%)\end{array}$ & $113(66.1 \%)$ & $292(55.2 \%)$ & $\begin{array}{l}1.58(1.10- \\
2.27)\end{array}$ & 0.01 & $\begin{array}{l}1.43(1.15- \\
3.22)\end{array}$ & 0.002 \\
\hline \multirow[t]{4}{*}{ BMI* } & Mean (sd) & $31.0(5.6)$ & $30.6(5.2)$ & $31.0(5.7)$ & & $<0.0001$ & & \\
\hline & $\begin{array}{l}\text { Normal (18.5- } \\
24.9)\end{array}$ & $\begin{array}{l}78 \\
(12.3 \%)\end{array}$ & $27(12.25)$ & $51(10.7 \%)$ & Reference & & - & \\
\hline & $\begin{array}{l}\text { Overweight (25- } \\
29.9)\end{array}$ & $\begin{array}{l}262 \\
(41.4 \%)\end{array}$ & $75(47.8 \%)$ & $187(39.3 \%)$ & $\begin{array}{l}0.71(0.49- \\
1.02)\end{array}$ & 0.06 & $\begin{array}{l}1.1(0.85- \\
1.67)\end{array}$ & 0.20 \\
\hline & Obese $(\geq 30)$ & $\begin{array}{l}293 \\
(46.3 \%)\end{array}$ & $55(35.0 \%)$ & $238(50.0 \%)$ & $\begin{array}{l}1.86(1.28- \\
2.70)\end{array}$ & 0.001 & $1.32(1.1-2.3)$ & 0.001 \\
\hline \multicolumn{2}{|c|}{ Current smoker } & $\begin{array}{l}398 \\
(56.9 \%)\end{array}$ & $102(59.6 \%)$ & $296(56.0 \%)$ & $\begin{array}{l}0.86(0.61- \\
1.22)\end{array}$ & 0.40 & $\begin{array}{l}0.88(0.51- \\
1.50)\end{array}$ & 0.63 \\
\hline \multicolumn{2}{|c|}{ Current alcohol user } & $\begin{array}{l}157 \\
(22.4 \%)\end{array}$ & $54(31.6 \%)$ & $103(19.5 \%)$ & $\begin{array}{l}0.52(0.36- \\
0.77)\end{array}$ & 0.001 & $\begin{array}{l}0.72(0.38- \\
1.35)\end{array}$ & 0.30 \\
\hline \multirow{5}{*}{$\begin{array}{l}\text { Duration of } \\
\text { diabetes }\end{array}$} & Mean (sd) & $7.7(7.6)$ & $5.7(6.2)$ & $8.3(7.9)$ & & 0.07 & & \\
\hline & $<1 \mathrm{y}$ & $\begin{array}{l}108 \\
(15.7 \%)\end{array}$ & $29(17 \%)$ & $81(15.3 \%)$ & $\begin{array}{l}0.89(0.56- \\
1.41)\end{array}$ & 0.56 & $\begin{array}{l}0.59(0.36- \\
1.01)\end{array}$ & 0.28 \\
\hline & $1-5 y$ & $\begin{array}{l}239 \\
(34.8 \%)\end{array}$ & $62(36.7 \%)$ & $177(34.2 \%)$ & $\begin{array}{l}0.90(0.62- \\
1.29)\end{array}$ & 0.55 & $\begin{array}{l}0.69(0.45- \\
1.21)\end{array}$ & 0.89 \\
\hline & $6-10 y$ & $\begin{array}{l}166 \\
(24.2 \%)\end{array}$ & $40(23.7 \%)$ & $126(24.3 \%)$ & $\begin{array}{l}1.04(0.69- \\
1.56)\end{array}$ & 0.86 & $\begin{array}{l}0.65(0.58- \\
1.41)\end{array}$ & 0.96 \\
\hline & $>10 y$ & $\begin{array}{l}174 \\
(25.3 \%)\end{array}$ & $40(23.4 \%)$ & $134(25.3 \%)$ & $\begin{array}{l}2.08(0.84- \\
1.89)\end{array}$ & 0.25 & $\begin{array}{l}3.21(0.56- \\
4.41)\end{array}$ & 0.09 \\
\hline Dyslipidemia & Yes & $\begin{array}{l}430 \\
(61.4 \%)\end{array}$ & $71(17.5 \%)$ & $359(75.6 \%)$ & $\begin{array}{l}2.97(2.09- \\
2.54)\end{array}$ & 0.001 & $\begin{array}{l}4.79(3.43- \\
6.68)\end{array}$ & 0.01 \\
\hline
\end{tabular}

*BMI: body mass index

\section{Blood pressure control subgroup analysis}

Table 2 summarizes the demographic and clinical characteristics of hypertensive patients included in the study, as stratified by BP control status. Nighty-three patients $(20 \%)$ attained BP control, whereas the remaining 372 patients did not attain BP control (80\%). The uncontrolled BP group was found to be significantly older (mean $=62.1 \pm 11.0 \mathrm{y}$-old) as compared to the controlled BP one (mean $=57.2 \pm 11.1 \mathrm{y}$-old, $\mathrm{p}$-value $<0.001$ ). Similarly, the uncontrolled BP patients were more likely to be males $(\mathrm{OR}=5.03,95 \%$ CI: 2.86 8.86; p-value $<0.001)$. There was no statistically significant difference in the BMI between the BP uncontrolled group and the controlled one. Although smokers and alcohol consumers were less likely to be uncontrolled, the association was not statistically significant ( $p$-value>0.05). Similarly, diabetes-related information was not statistically different between the controlled and uncontrolled BP groups and these included the family history of DM, duration of DM and comorbid conditions. Patients diagnosed with isolated systolic hypertension (SBP) were more prone to be uncontrolled (OR $=2.85,95 \%$ CI: $1.26-6.42$, p-value $<0.05)$.

Lab results of the patients included in this study, as stratified by the $\mathrm{BP}$ control status are presented in table 3 . The only variable that was found to be statistically associated with uncontrolled BP is HDL, where the uncontrolled BP group was more likely to be uncontrolled according to their HDL value $(\mathrm{OR}=2.85,95 \% \mathrm{CI}$ : $1.71-4.71$, pvalue $<0.05$ ). Although both FBS and HbA1C were not statistically different between the two groups, it was found that the uncontrolled BP group were more likely to have uncontrolled FBS $(\mathrm{OR}=1.55$, $95 \%$ CI: $0.95-2.53$, p-value $>0.05)$ and uncontrolled A1C $(\mathrm{OR}=1.53$,
95\% CI: 0.96-2.46, p-value >0.05). Similarly, the uncontrolled BP group were less likely to be controlled according to their lipid profile. However, this association was not significant: OR for patients having LDL $\geq 100 \mathrm{mg} / \mathrm{dl}$ was 1.34 (95\% CI: 0.69-2.58, p-value $>0.05$ ), OR for patients having TG $\geq 150 \mathrm{mg} / \mathrm{dl}$ was 1.09 (95\% CI: 0.69-1.72, p-value $>0.05$ ), and OR for patients having TC $\geq 200 \mathrm{mg} / \mathrm{dl}$ was 1.19 (95\% CI: 0.70-2.02, p-value $>0.05$ )

Table 4 summarizes details of the medications used by the study sample stratified according to BP control. There was no statistical difference between both groups concerning the type of antihypertensive, antiplatelet and lipid-lowering medications use. Although the number of antihypertensive medications used was not statistically different between the two groups, uncontrolled BP group were more prone to use a lower number of antihypertensive medications: OR $=0.99(95 \% \mathrm{CI}: 0.59-1.66, \mathrm{p}$-value $>0.05)$ for those using two antihypertensive medications versus $\mathrm{OR}=0.65(95 \% \mathrm{CI}$ : 0.4-1.06, p-value>0.05) for those using more than two antihypertensive medications. Something worth highlighting is the finding that patients using incretin mimetics (glucagon-like peptide1 (GLP-1) analogs) were less likely to have uncontrolled BP and this association was statistically significant $(\mathrm{OR}=0.30,95 \% \mathrm{CI}$ : 0.18 0.49; p-value $<0.005$ ).

Finally, after adjusting for all covariates, multivariate analysis showed that only three factors were significantly associated with uncontrolled BP: Age being $\geq 65$ y (OR $=1.96$ (95\% CI: 1.07-3.61, pvalue $=0.03)$; male gender $(\mathrm{OR}=2.57,95 \% \mathrm{CI}: 1.41-4.66$, $\mathrm{p}$-value $=$ 0.002); and uncontrolled HDL (OR $=1.58,95 \%$ CI: 1.33-2.01, pvalue $=0.03$ ). 
Table 2: Study sample demographic characteristics stratified according to BP control

\begin{tabular}{|c|c|c|c|c|c|c|c|c|}
\hline \multicolumn{2}{|l|}{ Variables } & \multirow{2}{*}{$\begin{array}{l}\text { Total } \\
\text { N (\%) } \\
\text { N = 465 }\end{array}$} & \multirow{2}{*}{$\begin{array}{l}\mathrm{BP} \\
\text { controlled } \\
\mathrm{N}(\%) \\
\mathrm{N}=93\end{array}$} & \multirow{2}{*}{$\begin{array}{l}\text { BP } \\
\text { uncontrolled } \\
N(\%) \\
N=372\end{array}$} & \multirow[t]{2}{*}{ OR $(95 \% \mathrm{CI})$} & \multirow{2}{*}{$\begin{array}{l}\text { P- } \\
\text { value }\end{array}$} & \multirow{2}{*}{$\begin{array}{l}\text { Adjusted OR } \\
(95 \%)\end{array}$} & \multirow{2}{*}{$\begin{array}{l}\text { P- } \\
\text { value }\end{array}$} \\
\hline & & & & & & & & \\
\hline \multirow[t]{2}{*}{ Age (years) } & Mean (sd) & $61.4(11.1)$ & $57.2(11.1)$ & $62.1(11.0)$ & & 0.001 & & \\
\hline & $\geq 65$ & $186(40.0 \%)$ & $16(17.2 \%)$ & $170(45.7 \%)$ & $\begin{array}{l}4.08(2.28- \\
7.20)\end{array}$ & 0.001 & $\begin{array}{l}1.96(1.07- \\
3.61)\end{array}$ & 0.03 \\
\hline Gender & Male & $214(54.0 \%)$ & $17(18.2 \%)$ & 197 (52.9\%) & $\begin{array}{l}5.03(2.86- \\
8.86)\end{array}$ & 0.001 & $\begin{array}{l}2.57(1.41- \\
4.66)\end{array}$ & 0.002 \\
\hline \multirow[t]{4}{*}{ BMI } & Mean (sd) & $31.0(5.6)$ & $30.6(5.2)$ & $31.0(5.7)$ & & 0.59 & & \\
\hline & Normal (18.5-24.9) & $46(11.0 \%)$ & $16(17.2 \%)$ & $30(8 \%)$ & Reference & & & \\
\hline & Overweight (25-29.9) & $164(39.1 \%)$ & $36(30.9 \%)$ & $128(34.4 \%)$ & $\begin{array}{l}1.9(0.93- \\
3.86)\end{array}$ & 0.007 & $\begin{array}{l}1.82(0.73- \\
4.54)\end{array}$ & 0.20 \\
\hline & Obese $(\geq 30)$ & 209 (49.9\%) & $50(38.7 \%)$ & $159(42.7 \%)$ & $\begin{array}{l}1.7(0.85- \\
3.30)\end{array}$ & 0.13 & $\begin{array}{l}1.26(0.53- \\
2.95)\end{array}$ & 0.60 \\
\hline Current smoker & & $263(56.6 \%)$ & $58(62.3 \%)$ & $205(55.1 \%)$ & $\begin{array}{l}0.74(0.46- \\
1.18)\end{array}$ & 0.21 & $\begin{array}{l}0.88(0.51- \\
1.50)\end{array}$ & 0.63 \\
\hline Current alcohol user & & 87 (18.7\%) & $20(21.5 \%)$ & $67(18.0 \%)$ & $\begin{array}{l}0.67(0.39- \\
1.22)\end{array}$ & 0.22 & $\begin{array}{l}0.72(0.38- \\
1.35)\end{array}$ & 0.30 \\
\hline $\begin{array}{l}\text { Isolated systolic } \\
\text { hypertension at } \\
\text { diagnosis }\end{array}$ & $\begin{array}{l}\text { Uncontrolled } \\
(\geq 140)\end{array}$ & $77(66.9 \%)$ & $7(11.8 \%)$ & $70(17.7 \%)$ & $\begin{array}{l}2.85(1.26- \\
6.42)\end{array}$ & $<0.01$ & $\begin{array}{l}1.96(0.96- \\
6.42)\end{array}$ & 0.09 \\
\hline $\begin{array}{l}\text { Family history of } \\
\text { diabetes }\end{array}$ & & $308(66.2 \%)$ & $58(62.3 \%)$ & $250(67.2 \%)$ & $\begin{array}{l}1.24(0.77- \\
1.98)\end{array}$ & 0.38 & $\begin{array}{l}0.95(0.54- \\
1.67)\end{array}$ & 0.86 \\
\hline \multirow[t]{5}{*}{ Duration of diabetes } & Mean (sd) & $8.6(8.1)$ & $7.0(8.0)$ & $8.9(8.0)$ & & 0.07 & & 0.07 \\
\hline & $<1 \mathrm{y}$ & $55(12.1 \%)$ & $15(16.1 \%)$ & $40(10.7 \%)$ & Reference & & - & \\
\hline & $1-5 y$ & $151(33.1 \%)$ & $36(38.7 \%)$ & $115(30.9 \%)$ & $\begin{array}{l}1.20(0.59- \\
2.42)\end{array}$ & 0.61 & $\begin{array}{l}0.94(0.41- \\
2.16)\end{array}$ & 0.89 \\
\hline & $6-10 y$ & $112(24.6 \%)$ & $22(23.6 \%)$ & $90(24.0 \%)$ & $\begin{array}{l}1.53(0.72- \\
3.26)\end{array}$ & 0.26 & $\begin{array}{l}1.02(0.43- \\
2.45)\end{array}$ & 0.96 \\
\hline & $>10 y$ & $138(30.3 \%)$ & $28(30.1 \%)$ & $110(24.2 \%)$ & $\begin{array}{l}1.47(0.71- \\
3.04)\end{array}$ & 0.29 & $\begin{array}{l}2.26(0.88- \\
5.80)\end{array}$ & 0.09 \\
\hline \multirow[t]{4}{*}{$\begin{array}{l}\text { Diabetic } \\
\text { complications }\end{array}$} & Macrovascular & $146(31.4 \%)$ & $32(34.4 \%)$ & $114(30.6 \%)$ & $\begin{array}{l}0.84(0.52- \\
1.38)\end{array}$ & 0.58 & $\begin{array}{l}0.86(0.49- \\
1.49)\end{array}$ & 0.58 \\
\hline & Neuropathy & $90(19.4 \%)$ & $10(10.7 \%)$ & $80(21.5 \%)$ & $\begin{array}{l}1.35(0.66- \\
2.76)\end{array}$ & 0.42 & $\begin{array}{l}1.13(0.66- \\
3.46)\end{array}$ & 0.42 \\
\hline & Retinopathy & $42(9.0 \%)$ & $7(7.5 \%)$ & $35(9.4 \%)$ & $\begin{array}{l}1.28(0.55- \\
2.97)\end{array}$ & 0.57 & $\begin{array}{l}1.20(0.45- \\
3.18)\end{array}$ & 0.71 \\
\hline & Proteinuria & $160(34.4 \%)$ & $30(32.2 \%)$ & $130(35 \%)$ & $\begin{array}{l}1.13(0.70- \\
1.83)\end{array}$ & 0.63 & $\begin{array}{l}1.40(0.78- \\
2.51)\end{array}$ & 0.26 \\
\hline \multirow{4}{*}{ complications } & 0 & $168(36.1 \%)$ & 37 (39.7\%) & $131(35.2 \%)$ & Reference & & - & \\
\hline & 1 & $183(39.4 \%)$ & $33(35.4 \%)$ & $150(40.3 \%)$ & $\begin{array}{l}1.28(0.76- \\
2.17)\end{array}$ & 0.35 & $\begin{array}{l}1.33(0.73- \\
2.43)\end{array}$ & 0.35 \\
\hline & 2 & $91(19.6 \%)$ & $16(17.2 \%)$ & 75 (20.2\%) & $\begin{array}{l}1.32(0.69- \\
2.54)\end{array}$ & 0.4 & $\begin{array}{l}1.55(0.71- \\
3.37)\end{array}$ & 0.27 \\
\hline & $>2$ & $23(5.0 \%)$ & $5(5.3 \%)$ & $18(4.8 \%)$ & $\begin{array}{l}1.02(0.35- \\
2.92)\end{array}$ & 0.98 & $\begin{array}{l}0.91(0.29- \\
2.89)\end{array}$ & 0.87 \\
\hline
\end{tabular}

*BMI: body mass index

Table 3: Study sample lab data stratified according to BP control

\begin{tabular}{|c|c|c|c|c|c|c|c|c|}
\hline \multicolumn{2}{|l|}{ Variables } & \multirow{2}{*}{$\begin{array}{l}\text { Total } \\
\text { N (\%) } \\
\mathrm{N}=465 \\
\end{array}$} & \multirow{2}{*}{$\begin{array}{l}\text { BP controlled } \\
N(\%) \\
N=93 \\
\end{array}$} & \multirow{2}{*}{$\begin{array}{l}\text { BP uncontrolled } \\
\mathrm{N}(\%) \\
\mathrm{N}=372 \\
\end{array}$} & \multirow[t]{2}{*}{ OR (95\% CI) } & \multirow[t]{2}{*}{ P-value } & \multirow[t]{2}{*}{$\begin{array}{l}\text { Adjusted OR } \\
(95 \%)\end{array}$} & \multirow[t]{2}{*}{$\begin{array}{l}P \text { - } \\
\text { value }\end{array}$} \\
\hline \multirow{3}{*}{ FBS (mmol/l) } & & & & & & & & \\
\hline & Mean (sd) & $9.86(4.3)$ & $9.44(4.75)$ & $9.93(4.2)$ & & 0.45 & & \\
\hline & $\geq 7.32 \mathrm{mmol} / \mathrm{l}$ & $238(69.8 \%)$ & $28(30.1 \%)$ & $210(56.5 \%)$ & $\begin{array}{l}1.55(0.95- \\
2.53)\end{array}$ & 0.08 & $\begin{array}{l}1.81(0.97- \\
3.39)\end{array}$ & 0.06 \\
\hline \multirow{2}{*}{$\begin{array}{l}\mathrm{HbA1C} \\
(\mathrm{mmol} / \mathrm{l})\end{array}$} & Mean (sd) & $67(2.8)$ & $65(3)$ & $67(2.8)$ & & 0.31 & & 0.31 \\
\hline & $\geq(63 \mathrm{mmol} / \mathrm{l})$ & $288(69.4 \%)$ & $65(70 \%)$ & $223(60 \%)$ & $\begin{array}{l}1.53(0.96- \\
2.46)\end{array}$ & 0.07 & $\begin{array}{l}1.43(0.80- \\
2.58)\end{array}$ & 0.23 \\
\hline \multirow{4}{*}{$\begin{array}{l}\text { LDL-C } \\
(\mathrm{mmol} / \mathrm{l})\end{array}$} & Mean (sd) & $2.730(0.99)$ & $2.676(0.938)$ & $2.738(1)$ & & 0.67 & & \\
\hline & $\begin{array}{l}<1.8102 \\
\mathrm{mmol} / \mathrm{l}\end{array}$ & $56(14.9 \%)$ & $17(18.3 \%)$ & $39(10.5 \%)$ & Reference & & - & \\
\hline & $\begin{array}{l}1.8102-2.56014 \\
\mathrm{mmol} / \mathrm{l}\end{array}$ & $130(34.5 \%)$ & $27(29 \%)$ & 103 (27.7\%) & $\begin{array}{l}0.94(0.57- \\
1.55)\end{array}$ & 0.8 & $\begin{array}{l}0.95(0.37- \\
2.44)\end{array}$ & 0.91 \\
\hline & $\geq 2.586 \mathrm{mmol} / \mathrm{l}$ & $191(50.7 \%)$ & $47(50.5 \%)$ & $144(38.7 \%)$ & $\begin{array}{l}1.34(0.69- \\
2.58)\end{array}$ & 0.39 & $\begin{array}{l}0.87(0.36- \\
2.11)\end{array}$ & 0.76 \\
\hline HDL-C & Mean (sd) & $1.132(0.310)$ & $1.029(0.284)$ & $1.148(0.312)$ & & 0.01 & & \\
\hline
\end{tabular}




\begin{tabular}{|c|c|c|c|c|c|c|c|c|}
\hline \multicolumn{9}{|l|}{$(\mathrm{mmol} / \mathrm{l})$} \\
\hline & Uncontrolled * & 203 (53.8\%) & $23(24.7 \%)$ & $180(48.4 \%)$ & $\begin{array}{l}2.85(1.71- \\
4.71)\end{array}$ & $<0.0001$ & $\begin{array}{l}1.58(1.33- \\
2.01)\end{array}$ & 0.05 \\
\hline \multirow[t]{2}{*}{$\mathrm{TG}(\mathrm{mmol} / \mathrm{l})$} & Mean (sd) & $2.290(1.80)$ & $2.80(3.067)$ & $2.210(1.5)$ & & 0.18 & & \\
\hline & $\begin{array}{l}\text { Uncontrolled } \\
(\geq 1.693)\end{array}$ & $223(58.1 \%)$ & $43(46.2 \%)$ & $180(48.4 \%)$ & $\begin{array}{l}1.09(0.69- \\
1.72)\end{array}$ & 0.71 & $\begin{array}{l}0.90(0.50- \\
1.62)\end{array}$ & 0.71 \\
\hline \multirow[t]{2}{*}{$\mathrm{TC}(\mathrm{mmol} / \mathrm{l})$} & Mean (sd) & $4.742(1.223)$ & $4.680(1.375)$ & $4.750(1.199)$ & & 0.80 & & \\
\hline & $\begin{array}{l}\text { Uncontrolled } \\
(\geq 5.172)\end{array}$ & $122(31.4 \%)$ & $22(23.6 \%)$ & $100(26.9 \%)$ & $\begin{array}{l}1.19(0.70- \\
2.02)\end{array}$ & 0.53 & $\begin{array}{l}0.97(0.52- \\
1.80)\end{array}$ & 0.92 \\
\hline
\end{tabular}

FBS: fasting blood sugar; HbA1C: glycated hemoglobin; LDL-C: low-density lipoprotein cholesterol; HDL-C: high-density lipoprotein cholesterol; TG: serum triglycerides; TC: total cholesterol; *Uncontrolled for men was at $<1.034 \mathrm{mmol} / \mathrm{l}$, whereas for women was at $<1.293 \mathrm{mmol} / \mathrm{l}$

Table 4: Drug treatment of the study sample stratified according to BP control

\begin{tabular}{|c|c|c|c|c|c|c|c|c|}
\hline \multicolumn{2}{|l|}{ Variables } & \multirow{2}{*}{$\begin{array}{l}\text { Total } \\
\text { N (\%) } \\
N=465\end{array}$} & \multirow{2}{*}{$\begin{array}{l}\text { BP } \\
\text { controlled } \\
N(\%) \\
N=93 \\
\end{array}$} & \multirow{2}{*}{$\begin{array}{l}\text { BP } \\
\text { uncontrolled } \\
\mathrm{N}(\%) \\
\mathrm{N}=372 \\
\end{array}$} & \multirow{2}{*}{$\begin{array}{l}\text { OR }(95 \% \\
\text { CI) }\end{array}$} & \multirow{2}{*}{$\begin{array}{l}P \text { - } \\
\text { value }\end{array}$} & \multirow{2}{*}{$\begin{array}{l}\text { Adjusted } \\
\text { OR } \\
(95 \%) \\
\end{array}$} & \multirow{2}{*}{$\begin{array}{l}\text { P- } \\
\text { value }\end{array}$} \\
\hline & & & & & & & & \\
\hline \multirow[t]{6}{*}{ Anti-hypertensive agents } & ACEIs & $\begin{array}{l}222 \\
(28.4 \%)\end{array}$ & $42(45 \%)$ & $180(48.4 \%)$ & $\begin{array}{l}1.14(0.72- \\
1.8)\end{array}$ & 0.58 & $\begin{array}{l}0.85(0.48- \\
1.51)\end{array}$ & 0.58 \\
\hline & ARBs & $\begin{array}{l}234 \\
(50.3 \%)\end{array}$ & $46(49.5 \%)$ & $188(50.5 \%)$ & $\begin{array}{l}1.16(0.74- \\
1.80)\end{array}$ & 0.53 & $\begin{array}{l}1.58(0.92- \\
2.69)\end{array}$ & 0.10 \\
\hline & BBs & $\begin{array}{l}230 \\
(49.5 \%)\end{array}$ & $48(51.6 \%)$ & $182(48.9 \%)$ & $\begin{array}{l}0.90(0.57- \\
1.41)\end{array}$ & 0.64 & $\begin{array}{l}1.31(0.77- \\
2.22)\end{array}$ & 0.33 \\
\hline & Diuretics** & $\begin{array}{l}187 \\
(40.2 \%)\end{array}$ & $33(35.5 \%)$ & $154(41.4 \%)$ & $\begin{array}{l}1.07(0.66- \\
1.74)\end{array}$ & 0.78 & $\begin{array}{l}1.23(0.71- \\
2.13)\end{array}$ & 0.45 \\
\hline & CCBs & $\begin{array}{l}125 \\
(26.9 \%)\end{array}$ & $20(21.5 \%)$ & $105(28.2 \%)$ & $\begin{array}{l}1.44(0.83- \\
2.47)\end{array}$ & 0.19 & $\begin{array}{l}1.24(0.67- \\
2.29)\end{array}$ & 0.50 \\
\hline & Alpha-2 agonist & $9(1.9 \%)$ & $1(1 \%)$ & $8(2.1 \%)$ & $\begin{array}{l}2.02(0.25- \\
6.37)\end{array}$ & 0.50 & $\begin{array}{l}1.28(0.16- \\
10.43)\end{array}$ & 0.82 \\
\hline \multirow[t]{3}{*}{$\begin{array}{l}\text { Number of anti- } \\
\text { hypertensive medications }\end{array}$} & 1 & $\begin{array}{l}172 \\
(37.0 \%)\end{array}$ & $37(39.8 \%)$ & $137(36.8 \%)$ & Reference & & - & \\
\hline & 2 & $\begin{array}{l}166 \\
(35.7 \%)\end{array}$ & $36(38.7 \%)$ & $130(34.9 \%)$ & $\begin{array}{l}0.99(0.59- \\
1.66)\end{array}$ & 0.97 & $\begin{array}{l}1.80(0.56- \\
2.08)\end{array}$ & 0.90 \\
\hline & $>2$ & $\begin{array}{l}127 \\
(27.3 \%)\end{array}$ & $32(34.4 \%)$ & $95(25.5 \%)$ & $\begin{array}{l}0.65(0.4- \\
1.06)\end{array}$ & 0.09 & $\begin{array}{l}0.74(0.43- \\
1.26)\end{array}$ & 0.26 \\
\hline Statins & & $\begin{array}{l}267 \\
(57.4 \%)\end{array}$ & $55(59.1 \%)$ & $212(57 \%)$ & $\begin{array}{l}0.92(0.58- \\
1.45)\end{array}$ & 0.71 & $\begin{array}{l}1.14(0.67- \\
1.93)\end{array}$ & 0.63 \\
\hline \multirow[t]{3}{*}{ Anti-platelet medications } & Aspirin & $\begin{array}{l}217 \\
(46.7 \%)\end{array}$ & $39(41.9 \%)$ & $178(47.8 \%)$ & $\begin{array}{l}1.27(0.80- \\
2.01)\end{array}$ & 0.82 & $\begin{array}{l}1.07(0.63- \\
1.81)\end{array}$ & 0.82 \\
\hline & Clopidogrel & $\begin{array}{l}53 \\
(11.4 \%)\end{array}$ & $16(17.2 \%)$ & $37(9.9 \%)$ & $\begin{array}{l}0.59(0.31- \\
1.13)\end{array}$ & 0.11 & $\begin{array}{l}0.56(0.27- \\
1.16)\end{array}$ & 0.12 \\
\hline & $\begin{array}{l}\text { Aspirin } \\
+ \text { clopidogrel }\end{array}$ & $24(5.2 \%)$ & $4(3.1 \%)$ & $20(5.5 \%)$ & $\begin{array}{l}1.28(0.42- \\
3.19)\end{array}$ & 0.68 & $\begin{array}{l}1.80(0.41- \\
7.84)\end{array}$ & 0.43 \\
\hline \multirow[t]{4}{*}{$\begin{array}{l}\text { Anti-diabetic agents } \\
\text { (Non-insulin) }\end{array}$} & Metformin & $\begin{array}{l}348 \\
(74.8 \%)\end{array}$ & $68(73.1 \%)$ & $280(75.2 \%)$ & $\begin{array}{l}1.12(0.67- \\
1.87)\end{array}$ & 0.67 & $\begin{array}{l}0.99(0.54- \\
1.82)\end{array}$ & 0.97 \\
\hline & Sulphonylureas & $\begin{array}{l}218 \\
(46.9 \%)\end{array}$ & $39(42 \%)$ & $179(48.1 \%)$ & $\begin{array}{l}1.28(0.81- \\
2.03)\end{array}$ & 0.29 & $\begin{array}{l}1.08(0.63- \\
1.83)\end{array}$ & 0.79 \\
\hline & $\begin{array}{l}\text { Incretin } \\
\text { mimetics } * * *\end{array}$ & $\begin{array}{l}98 \\
(21.1 \%)\end{array}$ & $17(18.2 \%)$ & $81(21.7 \%)$ & $\begin{array}{l}0.30(0.18- \\
0.49)\end{array}$ & $<0.05$ & $\begin{array}{l}0.70(0.38- \\
1.28)\end{array}$ & 0.25 \\
\hline & Thiazolidinedione & $33(7.1 \%)$ & $4(4.3 \%)$ & $29(7.8 \%)$ & $\begin{array}{l}1.88(0.64- \\
5.49)\end{array}$ & 0.24 & $\begin{array}{l}1.17(0.40- \\
3.45)\end{array}$ & 0.78 \\
\hline Insulin alone & & $\begin{array}{l}96 \\
(20.6 \%)\end{array}$ & $20(21.5 \%)$ & $76(20.4 \%)$ & $\begin{array}{l}0.94(0.54- \\
1.63)\end{array}$ & 0.82 & $\begin{array}{l}1.47(0.62- \\
3.49)\end{array}$ & 0.29 \\
\hline Insulin+others & & $\begin{array}{l}65 \\
(14.0 \%)\end{array}$ & $17(18.3 \%)$ & $48(12.9 \%)$ & $\begin{array}{l}1.51(0.82- \\
2.77)\end{array}$ & 0.18 & $\begin{array}{l}1.38(0.60- \\
3.17)\end{array}$ & 0.45 \\
\hline
\end{tabular}

ACEIs: angiotensin converting enzyme inhibitors; ARB: angiotensin II receptor blockers; BB: beta blockers; CCBs: calcium channel blockers; *Those include acarbose or repaglinide; ${ }^{* *}$ Thiazides diuretics and spironlactone; ${ }^{* * *}$ Incretin mimetics include glucagon-like peptide-1 (GLP-1) analogs

\section{DISCUSSION}

Hypertension is a major cardiovascular risk factor for patients with T2DM especially if target BP is not reached. In this study, we assessed the prevalence of hypertension and BP control status among a cohort of T2DM Lebanese patientsas well as we identified the factors associated with being BP uncontrolled.

This study showed that hypertension is prevalent in $75 \%$ of T2DM patients. This result is similar to those reported in the literature where prevalence rates ranged from $60 \%$ to $80 \%$ depending on the sample size and patient's race $[1,20,21]$.
Moreover, this study highlighted that age older than $65 \mathrm{y}$, obesity, female gender as well as presence of dyslipidemia are factors that were associated with hypertension prevalence. These findings are in alignment with those reported in the literature where age, female gender, obesity and extensive atherosclerosis were reported as risk factors for hypertension development in T2DM patients [22].

The low prevalence of controlled BP among hypertensive T2DM patients found in this study (20\%) was comparable to findings in several studies reported in the literature. For example, in a study conducted between 2005 and 2006 in 26 countries, the overall BP control rate was $33.6 \%$ in men and $30.6 \%$ in women and was lower 
in diabetic as compared to non-diabetic patients [23]. Another study carried out in the United States by Grant et al. also reported similar prevalence of BP control (33\%) [24]. The low BP control rate among T2DM patients was reported to be related to the poor adherence to antihypertensive medications that has been reported to be as low as $43 \%$ [25-27]. Other reasons that might be implicated in poor BP control includes failure to adopt a healthy lifestyle (diet and exercise), underestimation of the potential complications of hypertension, absence of effective health education programs and low level of education and/or low socioeconomic levels [26-28]. Clinical inertia, which is the failure of health care providers to initiate or intensify therapy when indicated, was also reported as a key player that prevent BP goal attainment $[29,30]$.

On the other hand, other studies have reported a better prevalence of controlled BP as compared to our findings. For instance, two studies showed that around half of hypertensive diabetic patients are meeting target BP values [31, 32]. Barquilla et al. and Abougalambou et al. reported controlled BP rates in T2DM patients of $56.4 \%$, and $47.2 \%$ respectively $[33,34]$.

As for the factors associated with uncontrolled BPin our study, it was found that age $\geq 65 \mathrm{y}$ was associated with uncontrolled BP. Similar to our study, older age was significantly associated with uncontrolled BP as reported by Abougalambou et al. and by Duggirala et al. [34, 35].

Another factor found to significantly predict uncontrolled BP in our study was male gender $(\mathrm{OR}=2.57,95 \% \mathrm{CI}$ : 1.41-4.66, $\mathrm{p}$-value $=$ 0.002). Similar results were found in a study carried by Cummings $e t$ al. [36]. On the contrary Duggirala et al. reported that female gender was a significant predictor of uncontrolled BP control in their study [37]. Moreover, other studies reported that gender was not associated with uncontrolled BP [33, 34, 38, 39]. This can be explained by the fact that the mechanisms by which blood pressure increases in men and women with aging may be different after menopause [40].

On the other hand, we found that uncontrolled HDL in our study was associated with higher odds of having uncontrolled BP. This is in alignment with the theory that HDL is cardioprotectiveleading to a better control of BP [41].

Unpredictably, we did not find any statistical significance regarding association between uncontrolled blood pressure and elevated total cholesterol, elevated BMI as well as the number of antihypertensive medications. These findings were different from those reported by other studies. For instance, in a study carried out by barquilla et al. in 2014 reported a strong association between elevated total cholesterol and uncontrolled BP [33]. In a study carried out by Mubarak et al. in Jordan, it was reported that uncontrolled BP was positively associated with BMI ( $\mathrm{P}=.001)$ [38]. Basile et al. in 2013 reported that the administration of at least one antihypertensive medication is associated with better blood pressure control [42]. Finally, one important finding that worth being highlighted is that the use of incretin mimetics (glucagon-like peptide-1 (GLP-1) analogs) was associated with a higher prevalence of blood pressure control. However, this study failed to identify their use as an independent predictor of BP control. This may be due to the small sample size of patients receiving these medications or the presence of confounding variables that distorted the association between the use of these drugs and BP control. The positive effects of these drugs on lowering BP is reported in the literature [43, 44]. Therefore, this result should be investigated by $\mathrm{r}$ further studies.

\section{Strengths and limitations}

The results of this study should be evaluated in the light of its strengths and limitations. Being among few addressing this important topic in the Middle East is its main strength. One of the limitations of the study is its observational nature as well as the lack of follow-up of patients over time. A single visit was taken into consideration when assessing control of BP. This might have led to some bias in the estimation of the BP control level, since they were recorded at one point in time. Moreover, the ADA (less stringent) BP goals were used. This might have had also overestimated the percentage of patients at BP goals. In addition, this study did not look at factors that might have contributed to the low rates of controlled BP such as medication adherence, appropriateness of prescribed antihypertensive medications (type and dosage regimen) as well as clinical inertia. Therefore, further studies on this subject are required.

\section{CONCLUSION}

This study showed that more than three-fourth of hypertensive T2DM patients has their BP uncontrolled in Lebanon.

According to this study attention should be given to patients who are $\geq 65$-year-old, male patients and those having uncontrolled HDL levels. They should be selected for intervention programs that might improve their BP control. These intervention programs might include, but are not limited to, assessment of BP goals achievement and intensifying/ modifying antihypertensive medications accordingly at each clinic visit as well as educating patients on their disease status and related behavioral changes. These interventions could lead to better BP control rates, decreasing therefore the risk of macrovascular complications and minimizing the health care expenditures in a country where the health resources are limited.

Moreover, the finding of a possible positive association between the use of incretin mimetics and BP control highlights the promising role of these drugs in the management of T2DM patients and opens the door for the need of further studies in this regard.

\section{Funding}

There was no funding for this study.

\section{CONFLICT OF INTERESTS}

All authors declare that they have no conflict of interest.

\section{REFERENCES}

1. Colosia AD, Palencia R, Khan S. Prevalence of hypertension and obesity in patients with type 2 diabetes mellitus in observational studies: a systematic literature review. Diabetes Metab Syndr Obes: Targets Ther 2013;6:327-38.

2. Grossman E, Messerli F. Hypertension and diabetes. Adv Cardiol 2008;45:82-106.

3. Campbell N, Gilbert R, Leiter L, Larochelle $\mathrm{P}$, Tobe $\mathrm{S}$, Chockalingam A, et al. Hypertension in people with type 2 diabetes: Update on pharmacologic management. Canadian Family Physician 2011;57:997-1002, e347-53.

4. Song S, Hardisty C. Type 2 diabetes mellitus: a high-risk condition for cardiovascular disease irrespective of the different degrees of obesity. Q J Med 2008;101:875-9.

5. Estacio RO, Jeffers BW, Gifford N, Schrier RW. Effect of blood pressure control on diabetic microvascular complications in patients with hypertension and type 2 diabetes. Diabetes Care 2000;23 Suppl 2:54-64.

6. Cushman W, Evans G, Byington R, Goff D, Grimm R, Cutler J. Effects of intensive blood-pressure control in type 2 diabetes mellitus. N Engl J Med 2010;362:1575-85.

7. Mancia G, Laurent S, Agabiti-Roseic E, Ambrosionid E, Burniere M, Caulfieldf M, et al. Reappraisal of European guidelines on hypertension management: a European society of hypertension task force document. J Hypertens 2009;27:2121-58.

8. Lopez-Jaramillo P, Lopez-Lopez J, Lopez-Lopez C, RodriguezAlvarez M. The goal of blood pressure in the hypertensive patient with diabetes is defined: now the challenge is go from recommendations to practice. Diabetol Metab Syndr 2014;6:31.

9. Holman R, Paul S, Bethel M, Neil H, Matthews D. Long-term follow-up after tight control of blood pressure in type 2 diabetes. N Engl J Med 2008;359:1565-76.

10. The Action to Control Cardiovascular Risk in Diabetes Study Group. Effects of intensive blood pressure control in type 2 diabetes mellitus. N Engl J Med 2008;358:2545-59.

11. Hanson l, Zanchetti A, Carruthers S, Dahlof B, Elmfeldt D, Julius $\mathrm{S}$, et al. Effects of intensive blood pressure lowering and lowdose aspirin in patients with hypertension: principal results of the hypertension optimal treatment (HOT) randomized trial. Lancet 1998;351:1755-62.

12. UK Prospective diabetes study group. Tight blood pressure control and risk of macrovascular and microvascular complications in type 2 diabetes: UKPDS 38. Br Med J 1998;317:703-13. 
13. National Collaborating Centre for Chronic Conditions (UK). Type 2 Diabetes: National Clinical Guideline for Management in Primary and Secondary Care (Update). London: Royal College of Physicians (UK); 2008. Available from: http://www.ncbi.nlm.nih.gov/ books/NBK53885. [Last accessed on 06 May 2016]

14. American Diabetes Association. Standards of medical care in diabetes. Diabetes Care 2013;36 Suppl 1:11-66.

15. Bangalore S, Kumar S, Lobach I, Messerli F. Blood pressure targets in subjects with type 2 diabetes mellitus/impaired fasting glucose: observations from traditional and Bayesian random-effects meta-analyses of randomized trials. Circulation 2011;123:2799-810.

16. Chahoud J, Mrad J, Semaan A, Asmar R. Prevalence of diabetes mellitus among patients with essential arterial hypertension. J Med Liban 2015;63:74-80.

17. Costanian C, Benett C, Hawlla N, Assaad C, Sibaii A. Prevalence, correlates and management of type 2 diabetes mellitus in Lebanon: Findings from a national population-based study. Diabetes Res Clin Pract 2014;105:408-15.

18. World Health Organisation. Prevalence of diabetes in the WHO Eastern Mediterranean Region. Available from: Http:// www.who.int/diabetes/facts/world_figures/index2.html. [Last accessed on 06 May 2016]

19. Tohme RA, Jurjus AR, Estephan A. The prevalence of hypertension and its association with other cardiovascular disease risk factors in a representative sample of the Lebanese population. J Hum Hypertens 2005;19:861-8.

20. Khan NA, Venkatachalam VV, Alakhali KM, Alavudeen SS, Dhanapal CK, Ansari SMA. Therapeutic management of hypertension and hyperlipidemia in type2 diabetes mellitus patients in southwestern region of Saudi Arabia: a pharmacist perspective. Asian J Pharm Clin Res 2014;7:241-5.

21. Andayan TMI, Mohamed Ibrahim MI, Asdie AH. Assessing the impact of complications on the direct medical costs of type 2 diabetes mellitus outpatients. Int J Chem Pharm Res 2010;2:32-5.

22. Simonson D. Etiology and prevalence of hypertension in diabetic patients. Diabetes Care 1988;11:821-5.

23. Bell D. Treatment of diabetic hypertension. Diabetes Obes Metab 2009;11:433-44

24. Grant RW, Buse JB, Meigs JB. Quality of diabetes care in U. S. academic medical centers: low rates of medical regimen change. Diabetes Care 2005;28:337-442.

25. Erdine S. Compliance with the treatment of hypertension: the potential of combination therapy. J Clin Hypertens 2010;12:40-6.

26. Fung V, Huang J, Brand R, Newhouse J, Hsu J. Hypertension treatment in a medicare population: adherence and systolic blood pressure control. Clin Ther 2007;29:972-84.

27. Muszbek N, Brixner D, Benedict A, Keskinaslan A, Khan Z. The economic consequences of noncompliance in cardiovascular disease and related conditions: a literature review. Int J Clin Pract 2008;62:338-51.

28. Cheung B, Li C. Diabetes and hypertension: is there a common metabolic pathway? Curr Atheroscler Rep 2012;14:160-6.

29. Basile J. Clinical inertia and blood pressure goal attainment. J Clin Hypertens 2009;11(12, Suppl 1):5-12.

30. O'Connor P, Sperl-Hillen J, Johnson P, Rush W, Biltz G. Clinical inertia and outpatient medical errors. In: Henriksen K, Battles JB, Marks ES. editors. Advances in Patient Safety: From Research to Implementation (Volume 2: Concepts and Methodology). Rockville (MD): Agency for Healthcare Research and Quality (US); 2005. p. 293-308.
31. Cummings D, Doherty L, Howard G, Howard V, Safford M, Prince V, et al. Blood pressure control in diabetes: temporal progress yet persistent racial disparities: national results from the $\mathrm{R}$ Easons for geographic and racial differences in stroke (REGARDS) study. Diabetes Care 2010;33:798-803.

32. Egan B, Zhao Y, Axon R. US trends in prevalence, awareness, treatment, and control of hypertension, 1988-2008. JAMA 2010;303:2043-50.

33. Barqilla G, listerri J, Prieto M, Alonso-Moreno F, Matarin G, Nafria G, et al. Blood pressure control in a population of hypertensive diabetic patients treated in primary care: PRESCAP-diabetes Study 2010. SEMERGEN-Med Familia 2015;41:13-23.

34. Abougalambou S, Abougalambou A. A study evaluating prevalence of hypertension and risk factors affecting on blood pressure control among type 2 diabetes patients attending teaching hospital in Malaysia. Diabetol Metab Syndr 2013;7:83-6.

35. Duggirala M, Cuddihy R, Cuddihy $M$, Naessens J, Cha $\mathrm{S}$, Mandrekar J, et al. Predictors of blood pressure control in patients with diabetes and hypertension seen in primary care clinics. Am J Hypertens 2005;18:833-8.

36. Cummings D, Letter A, Howard G, Howard V, Safford M, Prince $\mathrm{V}$, et al. Generic medications and blood pressure control in diabetic hypertensive subjects: results from the $\mathrm{R}$ easons for geographic and racial differences in stroke (REGARDS) study. Diabetes Care 2013;36:591-7.

37. Duggirala MK, Cuddihy RM, Cuddihy MT, Nyman M, Naessens JM Pankratz VS. Women with diabetes have poorer control of blood pressure than men. Int J Women's Health 2005;14:418-23.

38. Mubarak F, Froelicher E, Jaddou H, Ajlounia K. Hypertension among 1000 patients with type 2 diabetes attending a national diabetes center in Jordan. Ann Saudi Med 2008;28:346-51.

39. Serra N, Oliveras A, Bergoñon S, Sans L, Cobos A, Martínez P, et al. Factors associated with blood pressure control in diabetic patients treated in nephrology units: PRESDIAB study. Nefrologia 2011;31:313-21.

40. Lopez-Ruiz A, Sartori-Valinotti J, Yanes L, Iliescu R, Reckelhoff J. Sex differences in control of blood pressure: role of oxidative stress in hypertension in females. Am J Physiol Heart Circ Physiol 2008;295:466-74.

41. Miao D, YE P, Xiao E, Gao P, Zhang J, Wu HM. Influence of low high-density lipoprotein cholesterol on arterial stiffening and left ventricular diastolic dysfunction in essential hypertension. J Clin Hypertens 2011;13:710-5.

42. Basile J, Bloch M. Analysis of recent papers in hypertension: nighttime administration of at least one antihypertensive medication is associated with better blood pressure control and cardiovascular outcomes in patients with type 2 diabetes or chronic kidney disease. J Clin Hypertens 2013;15:2-4.

43. Katout M, Zhu H, Rutsky J, Shah P, Brook RD, Zhong J, et al. Effect of GLP-1 mimetics on blood pressure and relationship to weight loss and glycemia-lowering: results of a systematic meta-analysis and meta-regression. Am J Hypertens 2014;27:130-9.

44. Wang B, Zhong J, Lin $\mathrm{H}$, Zhao Z, Yan $\mathrm{Z}$, He $\mathrm{H}$, et al. Bloodpressure-lowering effects of GLP-1 receptor agonists exenatide and liraglutide: a meta-analysis of clinical trials. Diabetes Obes Metab 2013;15:737-49.

\section{How to cite this article}

- Lama Soubra, Hanan Nureddin, Amal Galal Omar, Mounzer Saleh. Factors associated with hypertension prevalence and control among lebanese type 2 diabetic patients. Int J Pharm Pharm Sci 2016;8(10):153-159. 